\title{
Fair Comparison of Complexity between Multi-band CAP and DMT for Data Center Interconnects
}

\author{
J. L. WeI, ${ }^{1,2, *}$ C. SANChez, ${ }^{3}$ E. GIACOUMIDIS ${ }^{4}$ \\ ${ }^{1}$ Is now with Huawei Technologies Düsseldorf GmbH, European Research Center, Riesstrasse 25, 80992 München, Germany. \\ ${ }^{2}$ Was with ADVA Optical Networking SE, Märzenquelle 1-3, 98617 Meiningen, Germany. \\ ${ }^{3}$ Aston Institute of Photonic Technologies (AIPT), Aston University, Birmingham B4 7ET, UK. \\ ${ }^{4}$ Sch. Engineering \& Physical Sciences, Heriot-Watt University, Edingburgh, Scotland EH14 4AS, UK \\ *Corresponding author: iinlonq.wei@huawei.com
}

Received XX Month 2017; revised XX Month, 2017; accepted XX Month 2017; posted XX Month 2017 (Doc. ID XXXXX); published XX Month 2017

\begin{abstract}
We present the first known detailed analysis and fair comparison of complexity of 56-Gb/s Multi-band CAP and DMT over 80-km DCF-free SMF links based on intensity modulation and direct detection (IMDD) for data center interconnects. We show that the matched FIR filters and (I)FFT take the majority of the complexity of the Multiband CAP and DMT, respectively. Choice of the multiband CAP sub-band count and the DMT (I)FFT size makes significant impact to system complexity or performance and trade-off must be considered. (C) 2017 Optical Society of America
\end{abstract}

OCIS codes: (060.2330) Fiber optics communications; (060.4080) Modulation;

http://dx.doi.org/10.1364/OL.99.099999

The ubiquitous use of cloud services has triggered high speed optical links for data center interconnects at typically multiple 400 $\mathrm{Gb} / \mathrm{s}$ bit rate over a single fiber [1,2]. Advanced modulation formats in combination with coding and digital signal processing (DSP) are enabling technologies to handle such large amount of data traffic [1-3]. The connection distance required by inter-data center connects is typically up to $80 \mathrm{~km}$ single mode fiber (SMF). Coherent systems are technically viable to offer efficient data transmission with a single wavelength capacity up to 200/400 $\mathrm{Gb} / \mathrm{s}$ for such scenarios. Nevertheless, in the very near future, coherent solutions may not satisfy the stringent requirements on cost, power and footprint. Alternatively, direct detection (DD) schemes are potential low-cost solutions by up-scaling from and leveraging the ecosystem of short reach transceivers for a wavelength division multiplexing (WDM) link [1, 4-8]. Four level pulse amplitude modulation (PAM-4) [1, 4, 5], multi-band carrierless amplitude and phase modulation (CAP) $[1,6]$ (a variant of QAM scheme) and discrete multi-tone (DMT ) $[1,7,8]$ are the main schemes considered.

Demonstrations have shown that 56 or $112 \mathrm{~Gb} / \mathrm{s}$ PAM-4 signals can successfully transmit over $80-\mathrm{km}$ SMFs $[1,4,5]$. The advantage of PAM-4 is its simple implementation and the availability of the ready DSP technology [4]. However, PAM-4 has very limited dispersion tolerance thus a dispersion compensation fiber (DCF) is required to offset (partially) the fiber chromatic dispersion (CD) [1, 4]. Otherwise, optical vestigial sideband (VSB) PAM-4 can be incorporated to increase dispersion tolerance but the system requires very high optical signal to noise ratio (OSNR) [5]. Multi-band CAP or DMT using multiple bands/tones naturally have strong resilience to fiber $\mathrm{CD}$. Together with optical VSB via simple asymmetrical optical filtering, the two schemes have shown very little penalty after transmissions through $80-\mathrm{km}$ DCF-free SMFs and achieve reasonably high OSNR performance $[6,7,9]$ at $56 \mathrm{~Gb} / \mathrm{s}$ bit rate or more.

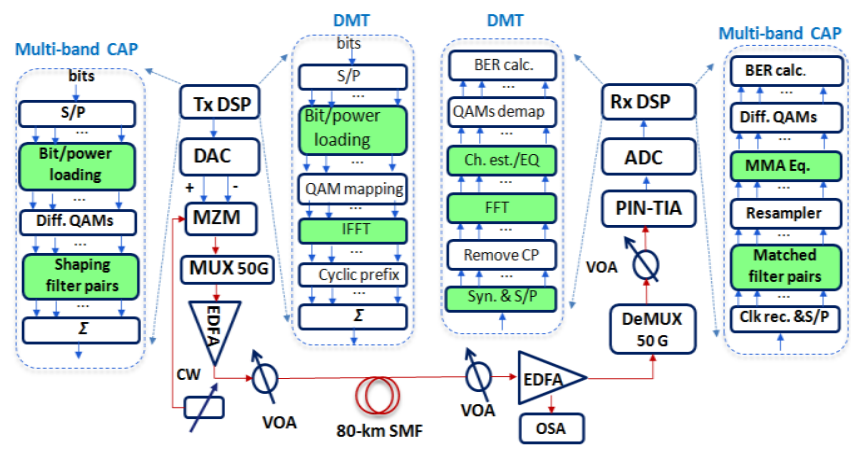

Fig. 1. Experimental setup for 56-Gb/s multi-band CAP and DMT systems. The DSP blocks with green background contribute the major complexity.

Previous demonstrations showed that under the same hardware components and setup, 56-Gb/s optical VSB multi-band CAP and DMT achieve similar OSNR performance [6, 7, 9]. Multiband CAP usually has a sub-band count much less than the (I)IFFT size of its counterpart DMT. One may take it for granted that multiCAP brings about less DSP complexity, which is an important concern regarding transceiver DSP power, compared to DMT. Although complexity comparison has been performed between 
CAP and DMT $[3,10]$, it was only based on single band CAP and 0band short reach scenarios considering no more than $10-\mathrm{km}$ distances. This paper dedicates to the first detailed analysis and fair comparison of the transceiver complexity of a $56 \mathrm{~Gb} / \mathrm{s}$ multiband CAP and DMT for $80-\mathrm{km}$ IMDD DCF-free SMF link. It will reveal the major parameters that impact the system complexity for both DD links and advise the important implementation rules.

Fig. 1 shows the experimental setup for $56 \mathrm{~Gb} / \mathrm{s}$ Multi-band CAP and DMT systems. It consists of transceiver DSPs and optics. The offline Tx DSP generates the wanted waveform which is then converted into an analogue signal via a digital to analogue convertor (DAC) operating at $80 \mathrm{GS} / \mathrm{s}(84 \mathrm{GS} / \mathrm{s}$ ) for Multi-band CAP (DMT). The DAC output directly drives a Mach-Zehnder modulator (MZM). A following multiplexer (MUX) with a 50-G DWDM grid and a 3-dB bandwidth of approximately $39 \mathrm{GHz}$ is adopted and its output is amplified by a booster erbium-doped fiber amplifier (EDFA) and adjusted by a variable optical attenuator (VOA). By tuning the wavelength of the laser a frequency offset between the laser frequency and the MUX center frequency is introduced leading to a VSB multi-band CAP or DMT signal. After transmission over a DCF-free 80-km SMF, a combined VOA and pre-amplifier EDFA is used to load optical noise onto the received signal. The resulting OSNR is measured by an optical spectrum analyzer (OSA) which is connected to the pre-amplifier. Then a 50-G de-multiplexer (De-MUX) further filters the optical signal and a VOA is used to optimize the input power injected into a 28-G PIN-TIA. The detected signal is then converted into a digital signal by an ADC with the same sampling rate of DAC and then undergoes offline signal processing.

The transmitter and receiver DSPs for Multi-band CAP are shown in Fig. 1(b). As detailed in [6], the multi-band CAP Tx DSP consists a few blocks and the major DSP comes from the power loading (PL), and square-root raised cosine (SRRC) pulse shaping for signal at each band. The Rx DSP complexity takes into account matched filter pairs as well as the modified multi-modulus algorithm (MMA)-based equalization. These blocks are filled with green background in Fig. 1 . Note that zero overhead signal recovery is achieved in multi-band CAP by combining MMA equalizer and partial differential coded QAM [6].

The transceiver DSP for DMT is depicted in Fig. 1(c) and its detailed explanations can be found in [7]. The major complexity comes from the transmitter PL and IFFT, as well as the receiver training symbol based frame synchronization and channel estimation, the FFT and one-tap equalizers. The cyclic prefix and training symbols induced overhead must be considered in the following complexity analysis.

Throughout this paper, the DSP complexity is measured as the required number of real-valued multiplications per second since multiplication arithmetic operation is most resource consuming. We assume one complex multiplication (division) needs four (six) real-valued multiplications.

For multi-band CAP, the transceiver complexity is mainly from the Tx PL which requires $2 * \mathrm{~N}$ multiplications and $\mathrm{N}$ is the subband count, the Tx shaping filter (Rx matched filter) with SRRC impulse response, and the Rx feedforward MMA equalizer (FFE). Since the Tx shaping filters can be implemented as look-up tables (LUTs) [11], we include only in our analysis the time domain matched FIR filters' complexity, which depends on two factors: the symbol count (L) and the required samples count $\left(\mathrm{M}_{\mathrm{k}}\right)$ per symbol for the k-th sub-band. According to Nyquist theory, we have
Table 1. SRRC impulse response oversampling requirements

\begin{tabular}{|c|c|c|c|}
\hline $\begin{array}{l}\text { Sub- } \\
\text { band } k\end{array}$ & $\begin{array}{c}\mathrm{BW}(\mathrm{GHz}) \\
k(1+\mathrm{a}) R_{\mathrm{s}}\end{array}$ & $\begin{array}{l}\text { Nyquist } \\
\text { sampl. rate }\end{array}$ & $\begin{array}{c}\text { Samples/symbol } \\
\mathrm{M}_{\mathrm{k}}\left(\mathrm{M}_{\mathrm{k}} \approx 2 \mathrm{k}+1\right)\end{array}$ \\
\hline 1 & 2.2 & 4.4 & 3 \\
\hline 2 & 4.4 & 8.8 & 5 \\
\hline 3 & 6.6 & 13.2 & 7 \\
\hline 4 & 8.8 & 17.6 & 9 \\
\hline 5 & 11 & 22 & 11 \\
\hline 6 & 13.2 & 26.4 & 14 \\
\hline 7 & 15.4 & 30.8 & 16 \\
\hline 8 & 17.6 & 35.2 & 18 \\
\hline 9 & 19.8 & 39.6 & 20 \\
\hline 10 & 22 & 44 & 22 \\
\hline 11 & 24.2 & 48.4 & 25 \\
\hline 12 & 26.4 & 52.8 & 27 \\
\hline$\Sigma \mathrm{M}_{\mathrm{k}}$ & \multicolumn{3}{|c}{$M_{k} R_{s} \geq(1+a) R_{s}$} \\
\hline
\end{tabular}

namely $M_{k} \geq \mathrm{k}(1+\mathrm{a})$ where $R_{s}$ is the symbol rate (which is 2 GBaud [6]) and $a$ is the roll-off coefficient of SRRC filters and a $=0.1$. In order to construct the SRRC filter, $M_{k}$ must be an integer. To reduce complexity we choose the minimum integer that satisfies Eq. (1) for the $k$-th sub-band and the overall required multiplication count for the matched filters (I and Q) is $\sum_{k=1}^{N} M_{k}$ per symbol. Table 1

lists the required $M_{k}$ for the adopted 12 sub-bands and the $k$-th sub-band SRRC impulse response waveform needs approximately $2 k+1$ samples/symbol. For the complex MMA FFE equalization, similarly, the multiplication count is $4^{*} \mathrm{~L}^{\prime *} \mathrm{~N}$ with $\mathrm{L}$ ' being the FFE tap count.

According to the above analysis, the complexity of the Multiband CAP can be expressed as

$$
C_{M C A P}=\left(2 N+2 \sum_{k=1}^{N} M_{k} L+4 L^{\prime} N\right) R_{s}
$$
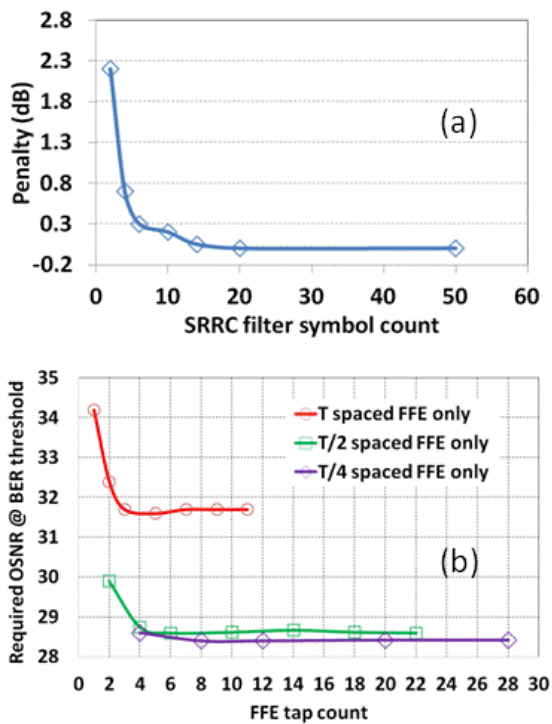

Fig. 2. (a) OSNR penalty @ BER = 3.8e-3 versus SSRC matched filter symbol count, and (b) required OSNR versus FFE tap count based on various tap spaces. 
It is now clear that Multi-CAP complexity is mainly dependent on the SRRC filter symbol count L and the FFE filter tap count L'. It is important to optimize the filters. Fig. 2(a) shows the OSNR penalty versus the receiver SRRC filter symbol count. The OSNR penalty decreases with increasing the SRRC filter symbol count. The penalty is negligible when the symbol count exceeds 10 . The complexity simply increases linearly with symbol count. Therefore, we choose $\mathrm{L}=10$ symbols for SRRC matched filters. Fig. 2(b) reflects the influence of the MMA FFE equalizer tap count on required OSNR @ BER = 3.8e-3. Three tap space cases, namely, T, $\mathrm{T} / 2$ and $\mathrm{T} / 4$ space are considered and $\mathrm{T}$ is the symbol time period. The penalty shows significant reduction with increasing the tap count regardless of tap space, and then begins to converge once tap count exceeds a certain value. The OSNR performance improves significantly when tap space reduces from $\mathrm{T}$ to $\mathrm{T} / 2 \mathrm{but}$ little change is observed when further reduces to $\mathrm{T} / 4$. Thus 4 taps $\mathrm{T} / 2$ space FFE filter can achieve optimum performance.

Similarly, the major DSP complexity for DMT is from the transmitter PL and IFFT and the receiver frame synchronization, channel estimation, FFT and one-tap equalizer. To achieve similar OSNR (about $28 \mathrm{~dB}$ ) performance to Multi-band CAP [6, 7] the required IFFT/FFT size is $\mathrm{N}=512$ [7]. In order to support antialiasing filtering, an oversampling factor of $f_{\mathrm{os}}=1.05$ is used meaning only $\mathrm{N} / 2 / \mathrm{f}_{\text {os }}$ subcarriers carry data. The VSB-DMT works in a training mode thus requires overhead. For an $80-\mathrm{km}$ SMF, the optimized training symbol overhead is $f_{\mathrm{tr}}=5 / 128$ meaning one DMT frame consists of 128 DMT symbols, 5 of which are training symbols. The optimized cyclic prefix sample count is Ncp $=32$ [7]. Within a DMT frame, the real multiplication count for transmitter $\mathrm{PL}$ and IFFT is $(128-5) * 2 * \mathrm{~N} / 2 / \mathrm{h}_{\mathrm{os}}$ and $128 * 2 \mathrm{~N} * \log _{2} \mathrm{~N}$ [3], respectively. The receiver FFT has the same complexity compared to IFFT and the one-tap equalizer needs $128 * 4 * \mathrm{~N} / 2 / \mathrm{h}_{\mathrm{os}} *\left(1-\mathrm{f}_{\mathrm{tr}}\right)$ real multiplications. Frame synchronization usually involves crosscorrelation, which can be implemented simply using adders since training symbols are known thus no multiplications are needed [12]. The channel estimation involves complex divisions in frequency domain needing $128^{*} 6^{*} \mathrm{~N}^{*} \mathrm{~h}_{\mathrm{tr}} / 2 / \mathrm{f}_{\mathrm{os}}$ real multiplications. by

In summary, the complexity of the DMT transceiver DSP is given

$$
C_{D M T}=\frac{\frac{123 N}{128 \eta_{o s}}+4 N \log _{2} N+\frac{3 N \cdot \eta_{t r}}{\eta_{o s}}+\frac{2 N\left(1-\eta_{t r}\right)}{\eta_{o s}}}{1 / f_{D A C} \cdot\left(N+N_{c p}\right)}
$$

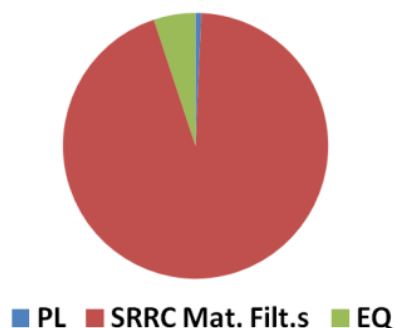

(a)

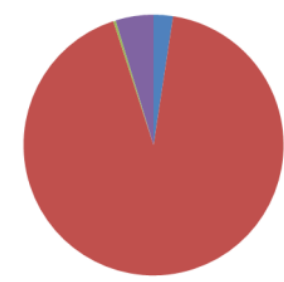

$\square \mathrm{PL} \square(\mathrm{I}) \mathrm{FFT} \backsim \mathrm{Ch}$. Est. $\square \mathrm{EQ}$

(b)
Fig. 3. Constituent DSP complexity of (a) Multi/band CAP, and (b) DMT.

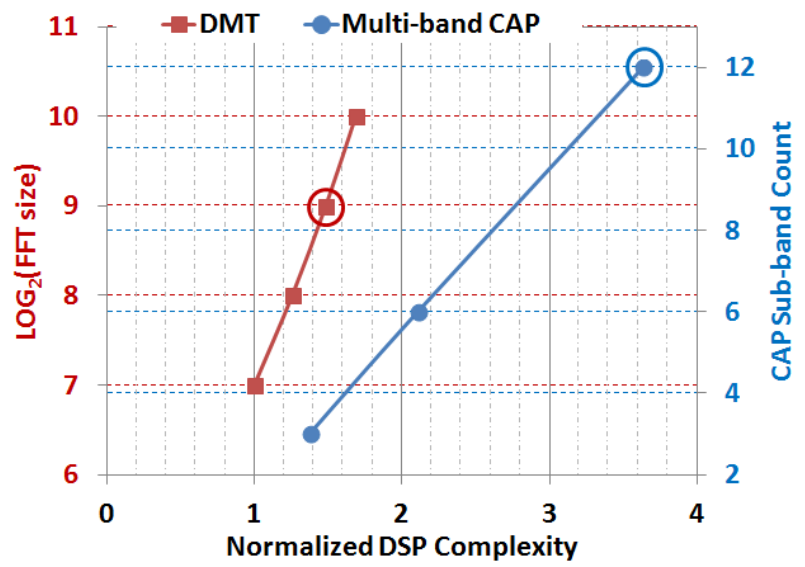

Fig. 4. Normalized DSP complexity dependence on (I)FFT size and subband count for DMT and multi-band CAP, respectively.

Based on the above analysis and Eqs. (2) and (3) as well as the optimized parameters, Fig. 3 shows the complexity distribution from constituent DSP parts. It clearly shows that receiver SRRC matched filters and transceiver (I)FFT take the majority complexity (over 90\%) for multi-band CAP and DMT, respectively. Therefore, simplifying SRRC filters and (I)FFT implementations are critical for multi-band CAP and DMT, respectively.

The complexity of the SRRC matched filters and (I)FFT is mainly dependent on the sub-band count and (I)FFT size, respectively. Fig. 4 reveals the relationship between the SRRC matched filter (IFFT) complexity and the CAP sub-band count (IFFT size). In obtaining Fig. 4, the other parameters other than $N$ shown in Eqs. (2) and (3) are assumed to be fixed, except that the multi-band CAP symbol rate changes in a way that its product with sub-band count is fixed in order to achieve a fixed bit rate. This is reasonable because the channel linear effects such as chromatic dispersion and component bandwidth limitations are very stable. Basically the complexity of DMT (Multi-band CAP) increases with increasing the FFT size (sub-band count), which is easy to understand via Eqs. (2) and (3). Another interesting phenomenon shown in Fig. 4 is that the complexity of Multi-band CAP shows much higher sensitivity to sub-band count than DMT complexity to FFT size. For example, the complexity of multi-band CAP almost halves when reducing the sub-band count from 12 to 6 . While DMT only shows $13 \%$ reduction in complexity when decreasing FFT size from 1024 to 512. This is mainly because the multi-band CAP complexity is proportional to the square of sub-band count, namely $O\left(N^{2}\right)$, as indicated by Eq. (2) by considering $\mathrm{M}_{\mathrm{k}} \approx 2 \mathrm{k}+1$ as shown in Table 1 . While DMT complexity is proportional to the logarithm of (I)FFT size $O\left(\log _{2} N\right)$. This indicates that (I)FFT implementation is more efficient than SRRC filters. Based on performance comparison made in [9] between optical VSB-based multi-band CAP and DMT, it shows that the two schemes have similar OSNR performance with a sub-band count of 12 and an (I)FFT size of 512, respectively. This means multi-band CAP requires about 2.4 times DSP complexity compared to DMT.

On the other hand, it is interesting to see from offline measurements that DMT shows $\sim 4-\mathrm{dB}$ OSNR penalty when reducing the (I)FFT size from 1024 to 128 and the performance tends to saturate when increasing (I)FFT size beyond 512 [7]. While for multi-band CAP, it is envisaged that a variation of subband count within 32 has not significant impact on the optical 
power (OSNR) sensitivity at BER threshold since it does not change much the signal properties such as peak to average ratio and the chromatic dispersion tolerance is similar since optical VSB is used. This tells us that for multi-band CAP systems, a smaller sub-band count is always preferred from complexity point of view, while for DMT, it is desirable to use moderate (I)FFT size to keep the best trade-off between complexity and OSNR performance. As a result, when the multi-band CAP system adopts a reduced subband count of 6 or 3, it shows comparable complexity to DMT.

To conclude, analysis and fair comparison of DSP complexity is conducted for $56 \mathrm{~Gb} / \mathrm{s}$ multi-band CAP and DMT over 80-km DCFfree SMFs. Results show that the time domain SRRC matched filters and (I)FFT take the majority of the complexity for multiband CAP and DMT, respectively. (I)FFT implementation is more efficient compared with time domain SRRC filters implementation from a complexity point of view. Multi-band CAP shows strong complexity sensitivity to the sub-band count thus a small sub-band is always preferred. DMT needs a moderate (I)FFT size to keep the best trade-off between complexity and OSNR performance. Analysis shows that the two schemes actually bring about comparable complexity with similar OSNR performance.

Funding. European Union Seventh Framework Programme (FP7) (623515); Horizon 2020 (659950).

Acknowledgment. We thank Dr. Annika Dochhan, Dr. Helmut Griesser, and Dr. Nicklas Eiselt from ADVA Optical Networking SE, Germany, for their support.

\section{References}

1. A. Dochhan, M. Eiselt, N. Eiselt, H. Griesser, J.-P. Elbers, J. J. Vegas Olmos, I.T. Monroy, in Proceedings of ECOC 2016 (2016), P680.

2. G. N. Liu, L. Zhang, T. Zuo, Q. Zhang, J. Zhou, and E. Zhou, in Proceeding of OFC 2017 (Optical Society of America, 2017), Th3D.5

3. J. Wei, Q. Cheng, R. Penty, I. H. White, and D. G. Cunningham, IEEE Commun. Magazin., 53, 182 (2015).

4. N. Eiselt, J. Wei, H. Griesser, A. Dochhan, M. H. Eiselt, J.-P. Elbers, J. J. V. Olmos, and I. T. Monroy, J. Lightw. Technol., 35, 955 (2017).

5. J. Lee, N. Kaneda, and Y-K. Chen, in Proceedings of ECOC 2016 (2016), P136.

6. J. Wei, N. Eiselt, C. Sanchez, R. Du, and H. Griesser, Opt. Lett., 41, 4122 (2016).

7. A. Dochhan, H. Griesser, N. Eiselt, M. Eiselt, J.-P. Elbers, in Proceedings of Photon. Netw. ITG-Symposium 2016 (VDE, 2016).

8. L. Zhang, T. Zuo, Q. Zhang, E. Zhou, G. N. Liu, and X. Xu, in Proceedings of ECOC 2015 (2015) We.4.6.4.

9. A. Dochan, N. Eiselt, J. Wei, H. Griesser, M. Eiselt, J. J. V. Olmos, I. T. Monroy, and J.-P.r Elbers, in proceedings of ACP 2016 (Optical Society of America, 2016), AS1B.3.

10. K. Zhong, X. Zhou, T. Gui, L. Tao, Y. Gao, W. Chen, J. Man, L. Zeng, A. P. T. Lau, C. Lu, Opt. Express, 23, 1176 (2015).

11. R. Schmogrow, R. Bouziane, M. Meyer, P. A. Milder, P. C. Schindler, R. I. Killey, P. Bayvel. C. Koos, W. Freude, and J. Leuthod, Opt. Express, 20, B543 (2012).

12. M. J. Canet, V. Almenar, S. J. Flores, J. Valls, J. Signal Process. Sys., 68, 287 (2012). 


\section{Full references}

1. A. Dochhan, M. Eiselt, N. Eiselt, H. Griesser, J.-P. Elbers, J. J. Vegas Olmos, I. T. Monroy, "Solutions for $400 \mathrm{Gbit} / \mathrm{s}$ Inter Data Center WDM Transmission," in Proceedings of ECOC 2016 (2016), P680.

2. G. N. Liu, L. Zhang, T. Zuo, Q. Zhang, J. Zhou, and E. Zhou, "IM/DD Transmission Techniques for Emerging $5 \mathrm{G}$ Fronthaul, $\mathrm{DCl}$ and Metro Applications," in Proceeding of OFC 2017 (Optical Society of America, 2017), Paper Th3D.5

3. J. Wei, Q. Cheng, R. Penty, I. H. White, and D. G. Cunningham, "400 Gigabit Ethernet Using Advanced Modulation Formats: Performance, Complexity, and Power Dissipation," IEEE Commun. Magazin., vol. 53, no. 2, p. 182 (2015).

4. N. Eiselt, J. Wei, H. Griesser, A. Dochhan, M. H. Eiselt, J.-P. Elbers, J. J. V. Olmos, and I. T. Monroy, "Evaluation of Real-Time $8 \times 56.25 \mathrm{~Gb} / \mathrm{s}$ PAM-4 for Inter-Data Center Application Over $80 \mathrm{~km}$ of SSMF at $1550 \mathrm{~nm}$," J. Lightw. Technol., Vol. 35, no. 4, p. 955 (2017).

5. J. Lee, N. Kaneda, and Y-K. Chen, "112-Gbit/s Intensity-Modulated DirectDetect Vestigial-Sideband PAM4 Transmission over an 80-km SSMF Link," Proc. ECOC, P136, Düsseldorf (2016).

6. J. Wei, N. Eiselt, C. Sanchez, R. Du, and H. Griesser, “ 56 Gb/s multi-band CAP for data center interconnects up to an $80 \mathrm{~km} \mathrm{SMF,"} \mathrm{Opt.} \mathrm{Lett.,} \mathrm{Vol.}$ 41, no. 17, p. 4122 (2016).

7. A. Dochhan, H. Griesser, N. Eiselt, M. Eiselt, J.-P. Elbers, “Optimizing Discrete Multi-tone Transmission for 400G Data Center Interconnects," in Proceedings of Photon. Netw. ITG-Symposium 2016 (2016).

8. L. Zhang, T. Zuo, Q. Zhang, E. Zhou, G. N. Liu, and X. Xu, "Transmission of 112-Gb/s+ DMT over 80-km SMF enabled by twin-SSB technique at 1550nm," ECOC, We.4.6.4, Valencia (2015).

9. A. Dochan, N. Eiselt, J. Wei, H. Griesser, M. Eiselt, J. J. V. Olmos, I. T. Monroy, and J.-P.r Elbers, "Practical Solutions for 400Gbit/s Data Center Transmission " in proceedings of ACP 2016, paper AS1B.3.

10. K. Zhong, X. Zhou, T. Gui, L. Tao, Y. Gao, W. Chen, J. Man, L. Zeng, A. P. T. Lau, C. Lu, "Experimental study of PAM-4, CAP-16, and DMT for $100 \mathrm{~Gb} / \mathrm{s}$ short reach optical transmission systems," Opt. Express, vol. 23, no. 2, p. 1176 (2015).

11. R. Schmogrow, R. Bouziane, M. Meyer, P. A. Milder, P. C. Schindler, R. I. Killey, P. Bayvel. C. Koos, W. Freude, and J. Leuthod, "Real-time OFDM or Nyquist pulse generation - which performs better with limited resources?" Opt. Express, vol. 20, no.26, p. B543 (2012).

12. M. J. Canet, V. Almenar, S. J. Flores, J. Valls, "Low Complexity Time Synchronization Algorithm for OFDM Systems with Repetitive Preambles," J. Signal Process. Sys., vol. 68, no. 3, p. 287 (2012). 